International Journal of Pure and Applied Mathematics

Volume 92 No. 5 2014, 777-784

ISSN: 1311-8080 (printed version); ISSN: 1314-3395 (on-line version)

url: http://www.ijpam.eu

doi: http://dx.doi.org/10.12732/ijpam.v92i5.11

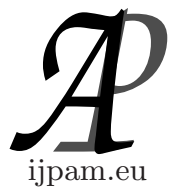

\title{
ON FAINTLY $g$-CONTINUOUS FUNCTIONS
}

\author{
N. Karthikeyan ${ }^{1}$ §, N. Rajesh ${ }^{2}$ \\ ${ }^{1}$ Department of Mathematics \\ Jeppiaar Engineering College \\ Chennai, 600119, Tamilnadu, INDIA \\ ${ }^{2}$ Department of Mathematics \\ Rajah Serfoji Govt. College \\ Thanjavur, 613005, Tamilnadu, INDIA
}

\begin{abstract}
A new class of functions called faintly $g$-continuous functions has been defined and studied in topological space. Also, the relationships between faintly $g$-continuous functions and graphs are investigated.
\end{abstract}

AMS Subject Classification: 54D10

Key Words: topological spaces, $g$-open sets, $g$-continuity, faintly $g$-continuity

\section{Introduction}

Generalized open sets play a very important role in General Topology and they are now the research topics of many topologists worldwide. Indeed a significant theme in General Topology and Real analysis concerns the variously modified forms of continuity, separation axioms etc. by utilizing generalized closed sets. In 1970, Levine [6] initiated the study of so-called $g$-closed sets, that is, a subset $A$ of a topological space $(X, \tau)$ is $g$-closed if the closure of $A$ included in every open superset of $A$ and defined a $T_{1 / 2}$ space to be one in which the closed sets and the $g$-closed sets coincide. In this paper, a new class of functions called

Received: October 4, 2013

(c) 2014 Academic Publications, Ltd.

$\S_{\text {Correspondence author }}$ url: www.acadpubl.eu 
faintly $g$-continuous functions has been defined and studied in topological space. Relationships among this new class of functions and $G O$-connected spaces and $G O$-compact spaces are investigated. Also, the relationship between faintly $g$-continuous functions and graphs are investigated.

\section{Preliminaries}

In the present paper, $(X, \tau)$ and $(Y, \sigma)$ represent topological spaces on which no separation axioms are assumed unless otherwise mentioned. For a subset $A$ of a space $(X, \tau), \operatorname{Cl}(A), \operatorname{Int}(A)$ and $A^{c}$ denote the closure of $A$, the interior of $A$ and the complement of $A$ in $X$, respectively. The complement of $g$-closed set is called $g$-open. The family of all $g$-open sets of $(X, \tau)$ is denoted by $G O(X)$. A point $x \in X$ is called a $\theta$-cluster point of $A$ if $\operatorname{Cl}(A) \cap A \neq \emptyset$ for every open set $V$ of $X$ containing $x$. The set of all $\theta$-cluster points of $A$ is called the $\theta$-closure of $A$ and is denoted by $\mathrm{Cl}_{\theta}(A)$. If $A=\mathrm{Cl}_{\theta}(A)$, then $A$ is said to be $\theta$-closed. The complement of $\theta$-closed set is said to be $\theta$-open. The union of all $\theta$-open sets contained in a subset $A$ is called the $\theta$-interior of $A$ and is denoted by $\operatorname{Int}_{\theta}(A)$. It follows from [12] that the collection of $\theta$-open sets in a topological space $(X, \tau)$ forms a topology $\tau_{\theta}$ on $X$.

Definition 1. A function $f:(X, \tau) \rightarrow(Y, \sigma)$ is said to be:

(i) faintly continuous [7] if $f^{-1}(V)$ is open in $X$ for every $\theta$-open set $V$ of $Y$.

(ii) $g$-continuous [1] if $f^{-1}(V)$ is $g$-closed set in $X$ for each closed set $V$ of $Y$.

(iii) $g c$-irresolute [1] if $f^{-1}(V)$ is $g$-closed set in $X$ for each $g$-closed set $V$ of $Y$.

(iv) $g c$-homeomorphism [8] if it is bijective, $g c$-irresolute and its inverse $f^{-1}$ is $g c$-irresolute.

(v) $g$-closed [9] if $f(F)$ is $g$-closed in $Y$ for every closed set $F$ of $X$.

\section{Faintly $g$-Continuous Functions}

Definition 2. A function $f:(X, \tau) \rightarrow(Y, \sigma)$ is said to be faintly $g$ continuous if $f^{-1}(V)$ is $g$-open in $X$ for every $\theta$-open set $V$ of $Y$. 
Theorem 3. For a function $f:(X, \tau) \rightarrow(Y, \sigma)$, the following statements are equivalent:

(i) $f$ is faintly $g$-continuous;

(ii) $f^{-1}(F)$ is $g$-closed in $X$ for every $\theta$-closed subset $F$ of $Y$;

(iii) $f:(X, \tau) \rightarrow\left(Y, \sigma_{\theta}\right)$ is $g$-continuous.

Proof. Easy proof and hence omitted.

Theorem 4. Every g-continuous function is faintly $g$-continuous.

Proof. Clear.

Remark 5. The converse of Theorem 4 is not true in general as can be seen from the following example.

Example 6. Let $X=\{a, b, c\}, \tau=\{\emptyset,\{a\}, X\}$ and $\sigma=\{\emptyset,\{b\},\{c\},\{b, c\}$, $X\}$. Then the identity function $f:(X, \tau) \rightarrow(X, \sigma)$ is faintly $g$-continuous but not $g$-continuous.

Definition 7. A topological space $(X, \tau)$ is said to be a $T_{1 / 2}$-space [6] if every $g$-closed subset of $(X, \tau)$ is in closed.

Theorem 8. Let $(Y, \sigma)$ be a $T_{1 / 2}$-space. Then a function $f:(X, \tau) \rightarrow$ $(Y, \sigma)$ is faintly $g$-continuous if and only if it is faintly continuous.

Proof. Follows from the Definition 7.

Theorem 9. If a function $f:(X, \tau) \rightarrow(Y, \sigma)$ is faintly g-continuous and $(Y, \sigma)$ is a regular space, then $f$ is $g$-continuous.

Proof. Let $V$ be any open set of $Y$. Since $Y$ is regular, $V$ is $\theta$-open in $Y$. Since $f$ is faintly $g$-continuous, by Theorem 3 , we have $f^{-1}(V)$ is $g$-open and hence $f$ is $g$-continuous.

Theorem 10. If a function $f:(X, \tau) \rightarrow(Y, \sigma)$ is faintly $g$-continuous, then for each point $x \in X$ and each $\theta$-open set $V$ containing $f(x)$, there exists a $g$-open set $U$ containing $x$ and $f(U) \subseteq V$.

Proof. Clear.

Remark 11. The following example shows that the composition of two faintly $g$-continuous functions need not be faintly $g$-continuous. 
Example 12. Let $X=\{a, b, c\}, \tau=\{\emptyset,\{a\}, X\}, \sigma=\{\emptyset,\{a\},\{b, c\}, X\}$ and $\eta=\{\emptyset,\{b\},\{a, c\}, X\}$. Define a function $f:(X, \tau) \rightarrow(X, \sigma)$ by $f(a)=$ $b, f(b)=a$ and $f(c)=c$ and $g:(X, \sigma) \rightarrow(X, \eta)$ an identity function. Then $f$ and $g$ are faintly $g$-continuous functions but their composition is not faintly $g$-continuous.

Now we investigate some basic properties of faintly $g$-continuous functions concerning composition and restriction. The proof of the first two results are straightforward and are omitted.

Theorem 13. If $f:(X, \tau) \rightarrow(Y, \sigma)$ is gc-irresolute and $g:(Y, \sigma) \rightarrow(Z, \eta)$ is faintly $g$-continuous, then $g \circ f:(X, \tau) \rightarrow(Z, \eta)$ is faintly $g$-continuous.

It is well known that a function $f:(X, \tau) \rightarrow(Y, \sigma)$ is said to be quasi- $\theta$ continuous [10] if $f^{-1}(V)$ is $\theta$-open in $(X, \tau)$ for every $\theta$-open set $V$ of $(Y, \sigma)$.

Theorem 14. If $f:(X, \tau) \rightarrow(Y, \sigma)$ is faintly $g$-continuous and $g$ : $(Y, \sigma) \rightarrow(Z, \gamma)$ is quasi- $\theta$-continuous, then $g \circ f:(X, \tau) \rightarrow(Z, \gamma)$ is faintly $g$-continuous.

Let $\left\{X_{\alpha}: \alpha \in \Lambda\right\}$ and $\left\{Y_{\alpha}: \alpha \in \Lambda\right\}$ be two families of topological spaces with the same index set $\Lambda$. The product space of $\left\{X_{\alpha}: \alpha \in \Lambda\right\}$ is denoted by $\Pi$ $\left\{X_{\alpha}: \alpha \in \Lambda\right\}$ (or simply $\Pi X_{\alpha}$ ). Let $f_{\alpha}: X_{\alpha} \rightarrow Y_{\alpha}$ be a function for each $\alpha \in \Lambda$. The product function $f: \Pi X_{\alpha} \rightarrow \Pi Y_{\alpha}$ is defined by $f\left(\left\{x_{\alpha}\right\}\right)=\left\{f_{\alpha}\left(x_{\alpha}\right)\right\}$ for each $\left\{x_{\alpha}\right\} \in \Pi X_{\alpha}$.

Theorem 15. If a function $f: X \rightarrow \Pi Y_{\alpha}$ is faintly $g$-continuous, then $P_{\alpha} \circ f: X \rightarrow Y_{\alpha}$ is faintly $g$-continuous for each $\alpha \in \Lambda$, where $P_{\alpha}$ is the projection of $\Pi Y_{\alpha}$ onto $Y_{\alpha}$.

Proof. Let $V_{\alpha}$ be any $\theta$-open set of $Y_{\alpha}$. Then, $P_{\alpha}^{-1}\left(V_{\alpha}\right)$ is $\theta$-open in $\Pi Y_{\alpha}$ and hence $\left(P_{\alpha} \circ f\right)^{-1}\left(V_{\alpha}\right)=f^{-1}\left(P_{\alpha}^{-1}\left(V_{\alpha}\right)\right)$ is $g$-open in $X$. Therefore, $P_{\alpha} \circ f$ is faintly $g$-continuous.

Theorem 16. Let $f:(X, \tau) \rightarrow(Y, \sigma)$ and $g:(Y, \sigma) \rightarrow(Z, \gamma)$ be functions. If $f$ is bijective, continuous and $g$-closed, and if $g \circ f:(X, \tau) \rightarrow(Z, \gamma)$ is faintly $g$-continuous, then $g:(Y, \sigma) \rightarrow(Z, \gamma)$ is faintly $g$-continuous.

Proof. Let $V$ be a $\theta$-open set of $Z$. Then $(g \circ f)^{-1}(V)=f^{-1}\left(g^{-1}(V)\right)$ is $g$-open in $X$. Since $f$ is bijective, continuous and $g$-closed, $f$ maps $g$-open sets to $g$-open sets [[3], Theorem 3] hence it follows that $g^{-1}(V)=f\left(f^{-1}\left(g^{-1}(V)\right)\right)$ is $g$-open in $Y$. 
Corollary 17. Let $f:(X, \tau) \rightarrow(Y, \sigma)$ be a bijective gc-homeomorphism and let $g:(Y, \sigma) \rightarrow(Z, \gamma)$ be a function. Then $g \circ f:(X, \tau) \rightarrow(Z, \gamma)$ is faintly $g$-continuous if and only if $g$ is faintly $g$-continuous.

Theorem 18. If $f:(X, \tau, \mathcal{I}) \rightarrow(Y, \sigma)$ is faintly g-continuous and $A$ is a closed subset of $X$, then $f_{\mid A}:\left(A, \tau_{\mid A}\right) \rightarrow(Y, \sigma)$ is faintly $g$-continuous.

Proof. The proof follows from Corollary 2.7 of [6].

Theorem 19. Let $f:(X, \tau) \rightarrow(Y, \sigma)$ be a function and $g:(X, \tau) \rightarrow$ $(X \times Y, \tau \times \sigma)$ the graph function of $f$, defined by $g(x)=(x, f(x))$ for every $x \in X$. If $g$ is faintly $g$-continuous, then $f$ is faintly $g$-continuous.

Proof. Let $U$ be an $\theta$-open set in $(Y, \sigma)$, then $X \times U$ is a $\theta$-open set in $X \times Y$. It follows that $f^{-1}(U)=g^{-1}(X \times U) \in G O(X)$. This shows that $f$ is faintly $g$-continuous.

Definition 20. A topological space $(X, \tau)$ is said to be $G O$-connected [1] if $X$ cannot be written as a disjoint union of two nonempty $g$-open sets.

Theorem 21. If $f:(X, \tau) \rightarrow(Y, \sigma)$ is a faintly $g$-continuous function and $(X, \tau)$ is a $G O$-connected space, then $Y$ is a connected space.

Proof. Assume that $(Y, \sigma)$ is not connected. Then there exist nonempty open sets $V_{1}$ and $V_{2}$ such that $V_{1} \cap V_{2}=\emptyset$ and $V_{1} \cup V_{2}=Y$. Hence we have $f^{-1}\left(V_{1}\right) \cap f^{-1}\left(V_{2}\right)=\emptyset$ and $f^{-1}\left(V_{1}\right) \cup f^{-1}\left(V_{2}\right)=X$. Since $f$ is surjective, $f^{-1}\left(V_{1}\right)$ and $f^{-1}\left(V_{2}\right)$ are nonempty subsets of $X$. Since $V_{i}$ is open and closed, $V_{i}$ is $\theta$-open for each $i=1,2$. Since $f$ is faintly $g$-continuous, $f^{-1}\left(V_{i}\right) \in G O(X)$. Therefore, $(X, \tau)$ is not $G O$-connected. This is a contradiction and hence $(Y, \sigma)$ is connected.

Definition 22. A space $(X, \tau)$ is said to be $G O$-compact [1] (resp. $\theta$ compact [11]) if each cover of $X$ by $g$-open (resp. $\theta$-open) has a finite subcover.

Theorem 23. The surjective faintly g-continuous image of a GO-compact space is $\theta$-compact.

Proof. Let $f:(X, \tau) \rightarrow(Y, \sigma)$ be a faintly $g$-continuous function from a $G O$-compact space $X$ onto a space $Y$. Let $\left\{G_{\alpha}: \alpha \in I\right\}$ be any $\theta$-open cover of $Y$. Since $f$ is faintly $g$-continuous, $\left\{f^{-1}\left(G_{\alpha}\right): \alpha \in I\right\}$ is a $g$-open cover of $X$. Since $X$ is $g$-compact, there exists a finite subcover $\left\{f^{-1}\left(G_{i}\right): i=1,2, \ldots\right.$. $n\}$ of $X$. Then it follows that $\left\{G_{i}: i=1,2, \ldots n\right\}$ is a finite subfamily which cover $Y$. Hence $Y$ is $\theta$-compact. 
Definition 24. A topological space $(X, \tau)$ is said to be:

(i) countably $g$-compact [5] (resp. countably $\theta$-compact) if every countable cover of $X$ by $g$-open (resp. $\theta$-open) sets has a finite subcover;

(ii) $g$-Lindelof $[5]$ (resp. $\theta$-Lindelof) if every cover of $X$ by $g$-open (resp. $\theta$-open) sets has a countable subocver.

Theorem 25. Let $f:(X, \tau) \rightarrow(Y, \sigma)$ be a faintly $g$-continuous surjective function. Then the following hold:

(i) If $X$ is $g$-Lindelof, then $Y$ is $\theta$-Lindelof;

(ii) If $X$ is countably $g$-compact, then $Y$ is countably $\theta$-compact.

Proof. The proof is similar to Theorem 23.

Definition 26. A topological space $(X, \tau)$ is said to be:

(i) $g-T_{1}[4]$ (resp. $\theta-T_{1}$ ) if for each pair of distinct points $x$ and $y$ of $X$, there exists $g$-open (resp. $\theta$-open) sets $U$ and $V$ containing $x$ and $y$, respectively such that $y \notin U$ and $x \notin V$.

(ii) $g-T_{2}[2]$ (resp. $\theta-T_{2}[11]$ ) if for each pair of distinct points $x$ and $y$ in $X$, there exists disjoint $g$-open (resp. $\theta$-open) sets $U$ and $V$ in $X$ such that $x \in U$ and $y \in V$.

Theorem 27. If $f:(X, \tau) \rightarrow(Y, \sigma)$ is faintly $g$-continuous injection and $Y$ is $\theta-T_{1}$, then $X$ is a $g-T_{1}$.

Proof. Suppose that $Y$ is $\theta-T_{1}$. For any distinct points $x$ and $y$ in $X$, there exist $V, W \in \sigma_{\theta}$ such that $f(x) \in V, f(y) \notin V, f(x) \notin W$ and $f(y) \in W$. Since $f$ is faintly $g$-continuous, $f^{-1}(V)$ and $f^{-1}(W)$ are $g$-open subsets of $(X, \tau)$ such that $x \in f^{-1}(V), y \notin f^{-1}(V), x \notin f^{-1}(W)$ and $y \in f^{-1}(W)$. This shows that $X$ is $g-T_{1}$.

Theorem 28. If $f:(X, \tau) \rightarrow(Y, \sigma)$ is faintly $g$-continuous injection and $Y$ is a $\theta-T_{2}$ space, then $X$ is a $g-T_{2}$ space.

Proof. Suppose that $Y$ is $\theta-T_{2}$. For any pair of distinct points $x$ and $y$ in $X$, there exist disjoint $\theta$-open sets $U$ and $V$ in $Y$ such that $f(x) \in U$ and $f(y) \in V$. Since $f$ is faintly $g$-continuous, $f^{-1}(U)$ and $f^{-1}(V)$ are $g$-open in $X$ containing $x$ and $y$, respectively. Therefore, $f^{-1}(U) \cap f^{-1}(V)=\emptyset$ because $U \cap V=\emptyset$. This shows that $X$ is $g-T_{2}$. 
Recall that for a function $f:(X, \tau) \rightarrow(Y, \sigma)$, the subset $\{(x, f(x)): x \in X\}$ $\subset X \times Y$ is called the graph of $f$ and is denoted by $G(f)$.

Theorem 29. If $f:(X, \tau) \rightarrow(Y, \sigma)$ is faintly $g$-continuous injection and $(Y, \sigma)$ is $\theta-T_{2}$, then $G(f)$ is $g$-closed in $X \times Y$.

Proof. Let $(x, y) \in(X \times Y) \backslash G(f)$, then $f(x) \neq y$. Since $Y$ is $\theta-T_{2}$, there exist $\theta$-open sets $V$ and $W$ in $Y$ such that $f(x) \in V, y \in W$ and $V \cap W=\emptyset$. Since $f$ is faintly $g$-continuous, $f^{-1}(V) \in G O(X, x)$. Take $U=f^{-1}(V)$. We have $f(U) \subset V$. Therefore, we obtain $f(U) \cap V=\emptyset$. This shows that $G(f)$ is $g$-closed.

Theorem 30. If $f:(X, \tau) \rightarrow(Y, \sigma)$ is a surjective function with a $g$-closed graph, then $(Y, \sigma)$ is Hausdorff.

Proof. Let $y_{1}$ and $y_{2}$ be any distinct points of $Y$. Then since $f$ is surjective, there exists $x_{1} \in X$ such that $f\left(x_{1}\right)=y_{1}$; hence $\left(x_{1}, y_{2}\right) \in(X \times Y) \backslash G(f)$. Since $G(f)$ is $g$-closed, there exist $U \in G O\left(X, x_{1}\right)$ and an open set $V$ of $Y$ containing $y_{2}$ such that $f(U) \cap \mathrm{Cl}(V)=\emptyset$. Therefore, we have $y_{1}=f\left(x_{1}\right) \in$ $f(U) \subset Y \backslash \mathrm{Cl}(V)$. Then there exists an open set $H$ of $Y$ such that $y_{1} \in H$ and $H \cap V=\emptyset$. Moreover, we have $y_{2} \in V$ and $V$ is open in $Y$. This shows that $Y$ is Hausdorff.

\section{References}

[1] K. Balachandran, P. Sundaram and H. Maki, On Generalized continuous functions in topological spaces, Mem. Fac. Sci. Kochi Univ., 12(1991), $5-13$.

[2] C. Boonpok, Preservation theorems concerning $g$-Hausdorff and $r g$ Hausdorff spaces, Naresuam Univ. J., 11(3)(2003), 75-77.

[3] M. Caldas, On g-closed sets and g-continuous functions, Kyungpook Math. J., 3(2)(1993), 205-209.

[4] M. Caldas and S. Jafari, On g-US spaces, Stus. Cerc. Mat., 14(2004), 13-20.

[5] A. Keskin and T. Noiri, Almost contra g-continuous functions, Kochi J. Math., 3(2008), 99-108.

[6] N. Levine, Generalized closed sets in topology, Rend. Cir. Mat. Palermo, 19(1970), 89-96. 
[7] P. E. Long and L. L. Herrington, The $T_{\theta}$-topology and faintly continuous functions, Kyungpook Math. J., 22(1982), 7-14.

[8] H. Maki, P. Sundaram and K. Balachandran, On generalized homeomorphism in topological spaces, Bull. Fukuoka Univ. Ed., III 40(1991), 13-21.

[9] S. R. Malgan, Generalized closed maps, J. Karnatak Univ. Sci., 27(82) (1982).

[10] T. Noiri and V. Popa, Weak forms of faint continuity, Bull. Math. Soc. Sci. Math. Roumanie, 34(82)(1990), 263-270.

[11] S. Sinharoy and S. Bandyopadhyay, On $\theta$-completely regular and locally Ө-H-closed spaces, Bull. Cal. Math. Soc., 87(1995), 19-26.

[12] N. V. Velicko, H-closed topological spaces, Amer. Math. Soc. Transl., 78(1968), 103-118. 